\title{
ON A ZONAL POLYNOMIAL INTEGRAL
}

\author{
A. K. GUPTA AND D. G. KABE
}

Received 2 September 2002 and in revised form 9 July 2003

A certain multiple integral occurring in the studies of Beherens-Fisher multivariate problem has been evaluated by Mathai et al. (1995) in terms of invariant polynomials. However, this paper explicitly evaluates the context integral in terms of zonal polynomials, thus establishing a relationship between zonal polynomial integrals and invariant polynomial integrals.

\section{Introduction}

Consider two $p \times p$ independent random matrices $S_{1}$ and $S_{2}$. The matrix $S_{1}$, with noncentrality parameter matrix $\Omega$ and covariance matrix $\Sigma$, is Wishart with $n_{1}$ degrees of freedom and $S_{2}$ is Wishart with covariance matrix $\Sigma$ and $n_{2}$ degrees of freedom. Then the random matrix $F=$ $S_{2}^{-1 / 2} S_{1} S_{2}^{-1 / 2}$ has the density

$$
\begin{aligned}
g(F)=K \int \mid & |F|^{(1 / 2)\left(n_{1}-p-1\right)}\left|S_{2}\right|^{(1 / 2)\left(n_{1}+n_{2}-p-1\right)} \\
& \times \exp \left\{-\frac{1}{2} \operatorname{tr} \Sigma_{2}^{-1} S_{2}-\frac{1}{2} \operatorname{tr} \Sigma_{1}^{-1} S_{2}^{1 / 2} F S_{2}^{1 / 2}\right\} \\
& \times{ }_{0} F_{1}\left(\frac{1}{2} n_{1} ; \frac{1}{4} \Omega \Sigma_{1}^{-1 / 2} S_{2}^{1 / 2} F S_{2}^{1 / 2}\right) d S_{2} \\
= & \sum_{\delta, \lambda: \phi} \frac{(-1)^{k} \theta_{\phi}^{\delta, \lambda} C_{\phi}(\Lambda)}{k ! \ell !\left(n_{1} / 2\right){ }_{\lambda} C_{\phi}(I)} \int C_{\phi}^{\delta, \lambda}\left(\frac{1}{2} \Sigma_{1}^{-1} S_{2}, \frac{1}{2} \Sigma_{1}^{-1} \Omega \Sigma_{1}^{-1 / 2} S_{2}\right) d S_{2},
\end{aligned}
$$


where $\Lambda$ is the $p \times p$ diagonal matrix of the roots of $F$ and $K$ as a generic letter denotes the normalizing constants of density functions in this paper.

Mathai et al. [4, equations (5.5.11), (5.5.12), pages 274-275] do not evaluate the integral (1.1). However, they derive the density of the roots of $F$ in terms of a series of invariant polynomials of two matrix arguments. This paper explicitly obtains the density of $F$ in terms of zonal polynomials, a result which has not been available in the literature so far, not even in terms of invariant polynomials. An expression for the density of the roots of $F$ is also derived in terms of invariant polynomials of two arguments, which is slightly different from the one presented by Mathai et al. [4, equation (5.5.11), page 274].

The next section states some useful results and the main results of the paper are derived in Section 3. Although it is not always possible to derive invariant polynomial results in terms of zonal polynomials, sometimes it is as done in this paper. We assume that all matrices occurring in this paper are full-rank matrices, except the noncentrality parameter $\Omega$ which may have any rank.

\section{Some useful results}

If $P$ is any $p \times p$ matrix, then it is known that (see Gupta and Nagar [2])

$$
K \int \exp \{\operatorname{tr} P V\}\left|I-V V^{\prime}\right|^{(1 / 2)(n-2 p-1)} d V={ }_{0} F_{1}\left(\frac{1}{2} n ; \frac{1}{4} P^{\prime} P\right) .
$$

Now we can write $P=\left(P^{\prime} P\right)^{1 / 2} H$ for some $p \times p$ orthogonal $H$, see G. A. Korn and T. M. Korn [3, page 412]. It follows that

$$
\begin{aligned}
& K \int \exp \{\operatorname{tr} Q P V\}\left|I-V V^{\prime}\right|^{(1 / 2)(n-2 p-1)} d V \\
& =K \int \exp \left\{\operatorname{tr} Q\left(P^{\prime} P\right)^{1 / 2} H V\right\}\left|I-V V^{\prime}\right|^{(1 / 2)(n-2 p-1)} d V \\
& =K \int \exp \left\{\operatorname{tr}\left(P^{\prime} P\right)^{1 / 2} Q^{\prime} V\right\}\left|I-V V^{\prime}\right|^{(1 / 2)(n-2 p-1)} d V \\
& ={ }_{0} F_{1}\left(\frac{1}{2} n ; \frac{1}{4} P^{\prime} P Q^{\prime} Q\right)={ }_{0} F_{1}\left(\frac{1}{2} n ; \frac{1}{4} Q^{\prime} Q P^{\prime} P\right) \\
& =K \int \exp \{\operatorname{tr} P Q V\}\left|I-V V^{\prime}\right|^{(1 / 2)(n-2 p-1)} d V .
\end{aligned}
$$


Again it follows from (2.2) that

$$
\begin{aligned}
K \int \exp \left\{\operatorname{tr} Q\left(P^{\prime} P\right)^{1 / 2} H V\right\}\left|I-V V^{\prime}\right|^{(1 / 2)(n-2 p-1)} d V \\
\quad=K \int \exp \left\{\operatorname{tr}\left(H^{\prime}\left(P^{\prime} P\right)^{1 / 2}\right) Q\right\}\left|I-V V^{\prime}\right|^{(1 / 2)(n-2 p-1)} d V \\
=K \int \exp \left\{\operatorname{tr}\left(H^{\prime}\left(P^{\prime} P\right)^{1 / 2}\right)\left(Q^{\prime} Q\right)^{1 / 2} R\right\}\left|I-V V^{\prime}\right|^{(1 / 2)(n-2 p-1)} d V \\
={ }_{0} F_{1}\left(\frac{1}{2} n ; \frac{1}{4} R^{\prime} Q^{\prime} Q R H^{\prime} P^{\prime} P H\right)={ }_{0} F_{1}\left(\frac{1}{2} n ; Q^{\prime} Q P^{\prime} P\right),
\end{aligned}
$$

where $R(p \times p)$ and $H(p \times p)$ are any two arbitrary orthogonal matrices which may depend on $Q^{\prime} Q$ and $P^{\prime} P$, respectively.

Now it follows from (2.3) that

$$
{ }_{0} F_{1}\left(\frac{1}{2} n ; \frac{1}{4} P^{\prime} P Q^{\prime} Q T^{\prime} T\right)={ }_{0} F_{1}\left(\frac{1}{2} n ; \frac{1}{4} H^{\prime} P^{\prime} P H R^{\prime} Q^{\prime} Q R U^{\prime} T^{\prime} T U\right),
$$

where $H, R$, and $U$ are any $p \times p$ orthogonal matrices, and note that

$$
{ }_{0} F_{1}\left(\frac{1}{2} n ; \frac{1}{4} A B C\right)={ }_{0} F_{1}\left(\frac{1}{2} n ; \frac{1}{4} \Delta\right) \text {, }
$$

where $\Delta$ is any permutation of $A B C$ and also holds for any permutation of four symmetric matrices.

Obviously, (2.4) in terms of zonal polynomials yields

$$
C_{\theta}\left(Q^{\prime} Q P^{\prime} P T^{\prime} T\right)=C_{\theta}\left(R^{\prime} Q^{\prime} Q R H^{\prime} P^{\prime} P H U^{\prime} T^{\prime} T U\right) .
$$

From (2.6), note that

$$
\exp \left\{\operatorname{tr} P^{\prime} P Q^{\prime} Q R^{\prime} R\right\}={ }_{0} F_{1} \exp \{\operatorname{tr} \Delta\}=\sum_{t=0}^{\infty} \frac{1}{t !} C_{\theta}\left(P^{\prime} P Q^{\prime} Q R^{\prime} R\right),
$$

where $\Delta$ is any permutation of $P^{\prime} P Q^{\prime} Q R^{\prime} R$. Further, it is easy to verify that

$$
{ }_{0} F_{1}\left(\frac{1}{2} n ; \frac{1}{4} P^{\prime} P Q^{\prime} Q T^{\prime} T G^{\prime} G\right)={ }_{0} F_{1}\left(\frac{1}{2} n ; \frac{1}{4} P^{\prime} P Q^{\prime} Q G^{\prime} G T^{\prime} T\right)
$$

because (2.8) is true for any permutation of the four distinct matrices in (2.8).

Now we proceed with the main result. 


\section{Main results}

In view of (2.4), (2.5), and (2.7) and using (1.1), the density of $F$ is given by

$$
\begin{aligned}
g(F)= & K|F|^{(1 / 2)\left(n_{1}-p-1\right)} \\
& \times \int \exp \left\{-\frac{1}{2} \operatorname{tr} \Sigma_{2}^{-1} S-\frac{1}{2} \operatorname{tr} \Sigma_{1}^{-1 / 2} F \Sigma_{1}^{-1 / 2} S\right\}|S|^{(1 / 2)\left(n_{1}+n_{2}-p-1\right)} \\
& \times{ }_{0} F_{1}\left(\frac{1}{2} n_{1} ; \frac{1}{4} \Sigma_{1}^{-1 / 2} \Omega \Sigma_{1}^{-1 / 2} F^{1 / 2} S F^{1 / 2}\right) d S \\
= & K|F|^{(1 / 2)\left(n_{1}-p-1\right)}\left|\Sigma_{2}^{-1}+\Sigma_{1}^{-1 / 2} F \Sigma_{1}^{-1 / 2}\right|^{-(1 / 2)\left(n_{1}+n_{2}\right)} \\
& \times{ }_{1} F_{1}\left(\frac{1}{2}\left(n_{1}+n_{2}\right), \frac{1}{2} n_{1} ;\right. \\
& \left.\quad \frac{1}{2} F^{1 / 2} \Sigma_{1}^{-1 / 2} \Omega \Sigma_{1}^{-1 / 2} F^{1 / 2}\left(\Sigma_{2}^{-1}+\Sigma_{1}^{-1 / 2} F \Sigma_{1}^{-1 / 2}\right)^{-1}\right) \\
& K|F|^{(1 / 2)\left(n_{1}-p-1\right)}\left|\Sigma_{2}^{-1}+\Sigma_{1}^{-1 / 2} F \Sigma_{1}^{-1 / 2}\right|^{-(1 / 2)\left(n_{1}+n_{2}\right)} \\
\times{ }_{1} F_{1}\left(\frac{1}{2}\left(n_{1}+n_{2}\right), \frac{1}{2} n_{1} ; \frac{1}{2}\left(\Sigma_{1}^{-1 / 2} \Omega \Sigma_{1}^{-1 / 2}\right)^{1 / 2}\right. & \left.\times F\left(\Sigma_{1}^{-1 / 2} \Omega \Sigma^{-1 / 2}\right)^{1 / 2}\left(\Sigma_{2}^{-1}+\Sigma_{1}^{-1 / 2} F \Sigma_{1}^{-1 / 2}\right)^{-1}\right)
\end{aligned}
$$

Now if $\Sigma_{1}^{1 / 2} \Sigma_{2}^{-1} \Sigma_{1}^{1 / 2}=\theta I$ for any scalar $\theta$, then note that

$$
\begin{aligned}
g(F)= & K|F|^{(1 / 2)\left(n_{1}-p-1\right)}|\theta I+F|^{-(1 / 2)\left(n_{1}+n_{2}\right)} \\
& \times{ }_{1} F_{1}\left(\frac{1}{2}\left(n_{1}+n_{2}\right), \frac{1}{2} n_{1} ; \frac{1}{2} \Omega F(\theta I+F)^{-1}\right) .
\end{aligned}
$$

Thus the density of $\Lambda=\operatorname{diag}\left(\lambda_{1}, \ldots, \lambda_{p}\right)$ of the roots of $F$ is

$$
\begin{aligned}
g(\Lambda)= & K|\Lambda|^{(1 / 2)\left(n_{1}-p-1\right)}|\theta I+\Lambda|^{-(1 / 2)\left(n_{1}+n_{2}\right)} \prod_{i<j}\left(\lambda_{i}-\lambda_{j}\right) \\
& \times{ }_{1} F_{1}\left(\frac{1}{2}\left(n_{1}+n_{2}\right), \frac{1}{2} n_{1} ; \frac{1}{2} \Omega \Lambda(\theta I+\Lambda)^{-1}\right) .
\end{aligned}
$$

The expression is not very suitable for obtaining the density of $\Lambda$ for the expression (3.1). For some $Q(p \times p)$ orthogonal, let $Q F Q^{\prime}$ be still denoted by $F$, let $L$ be the $p \times p$ diagonal matrix of the roots of $\Sigma^{1 / 2} \Omega \Sigma^{1 / 2}$, and let $\Delta$ be the diagonal matrix of the roots of $\Sigma_{1}^{1 / 2} \Sigma_{2}^{-1} \Sigma_{1}^{1 / 2}$. Then the 
density of this $F$ is

$$
\begin{aligned}
g(F)= & K|F|^{(1 / 2)\left(n_{1}-p-1\right)}|\Delta+F|^{-(1 / 2)\left(n_{1}+n_{2}\right)} \\
& \times{ }_{1} F_{1}\left(\frac{1}{2}\left(n_{1}+n_{2}\right), \frac{1}{2} n_{1} ; \frac{1}{2} L F(\Delta+F)^{-1}\right) .
\end{aligned}
$$

Mathai et al. [4, equation (A.3.5), page 338] showed that

$$
|\Delta+F|^{-a}{ }_{1} F_{1}\left(a, u ; L F(\Delta+F)^{-1}\right)=\sum_{\delta, \lambda ; \phi} \frac{(a)_{\phi} C_{\phi}^{\delta, \lambda}\left(-\Delta^{-1} F, L F\right)}{k K ! \ell !(u)_{\lambda}} .
$$

We have observed earlier that $C_{\theta}\left(Q^{\prime} Q P^{\prime} P\right)=C_{\theta}\left(R^{\prime} Q^{\prime} Q R H^{\prime} P^{\prime} P H\right)$ and thus the invariant polynomial argument for matrices can also be replaced by their roots. Thus the density of $\Lambda$ for (3.4) is

$$
\begin{aligned}
g(\Lambda)= & K|\Lambda|^{(1 / 2)\left(n_{1}-p-1\right)} \prod_{i<j}\left(\lambda_{i}-\lambda_{j}\right) \\
& \times \sum_{\delta, \lambda ; \phi} \frac{\left((1 / 2)\left(n_{1}+n_{2}\right)\right)_{\phi} C_{\phi}^{\delta, \lambda}\left(-\Delta^{-1} \Lambda, L \Lambda\right)}{k ! \ell !\left((1 / 2) n_{1}\right)_{\lambda}} .
\end{aligned}
$$

However, (3.6) is not suitable for obtaining the density of $(\operatorname{tr} F)$.

\section{Acknowledgment}

The authors are thankful to the referee for a critical reading of the paper.

\section{References}

[1] Y. Chikuse, Distributions of some matrix variates and latent roots in multivariate Behrens-Fisher discriminant analysis, Ann. Statist. 9 (1981), no. 2, 401-407.

[2] A. K. Gupta and D. K. Nagar, Matrix Variate Distributions, Chapman \& Hall/CRC Monographs and Surveys in Pure and Applied Mathematics, vol. 104, Chapman \& Hall/CRC, Boca Raton, Fla, USA, 2000.

[3] G. A. Korn and T. M. Korn, Mathematical Handbook for Scientists and Engineers, McGraw-Hill, New York, 1961.

[4] A. M. Mathai, S. B. Provost, and T. Hayakawa, Bilinear Forms and Zonal Polynomials, Lecture Notes in Statistics, vol. 102, Springer-Verlag, New York, 1995.

A. K. Gupta: Department of Mathematics and Statistics, Bowling Green State University, Bowling Green, OH 43403-0221, USA

E-mail address: gupta@bgnet.bgsu.edu

D. G. Kabe: Department of Mathematics and Computer Science, Saint Mary's University, Halefax, Nova Scotia, Canada B3H 3C3 


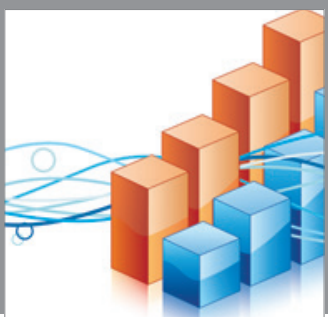

Advances in

Operations Research

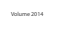

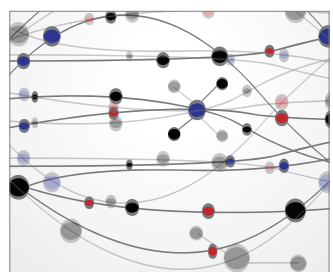

\section{The Scientific} World Journal
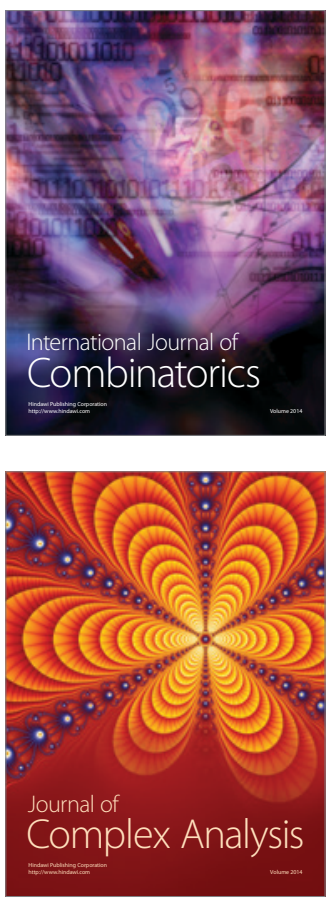

International Journal of

Mathematics and

Mathematical

Sciences
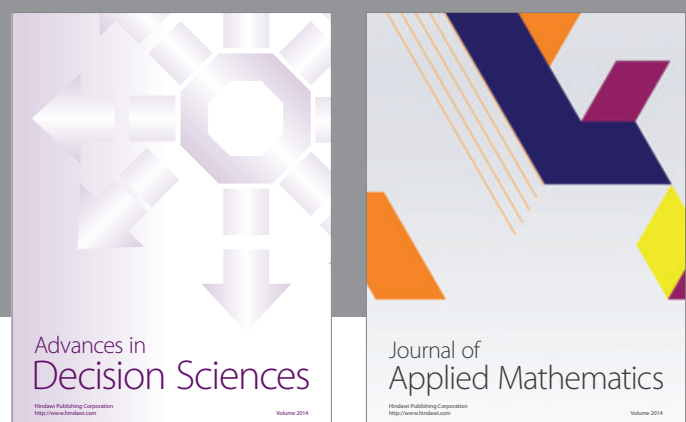

Journal of

Applied Mathematics
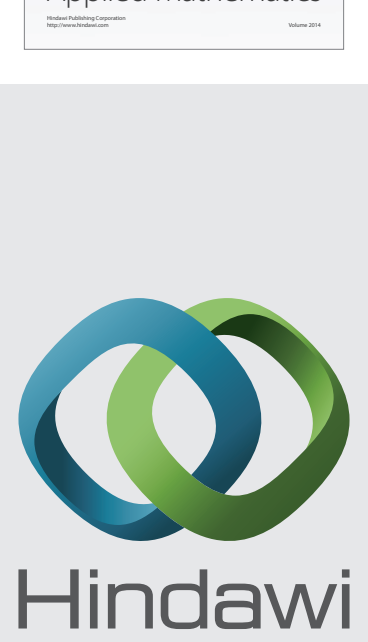

Submit your manuscripts at http://www.hindawi.com
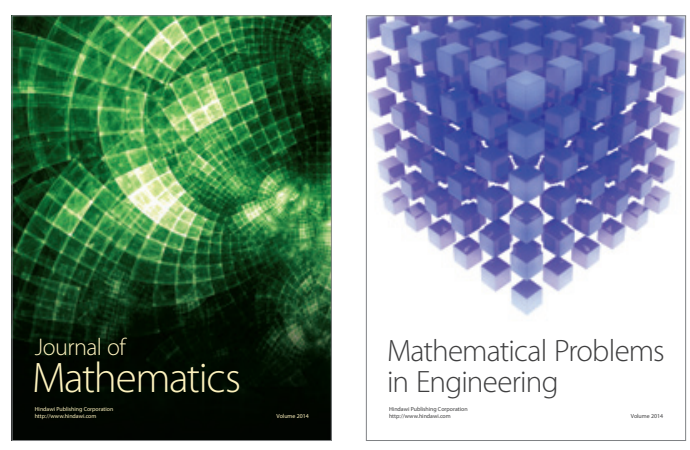

Mathematical Problems in Engineering
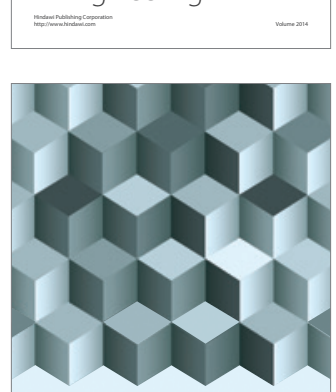

Journal of

Function Spaces
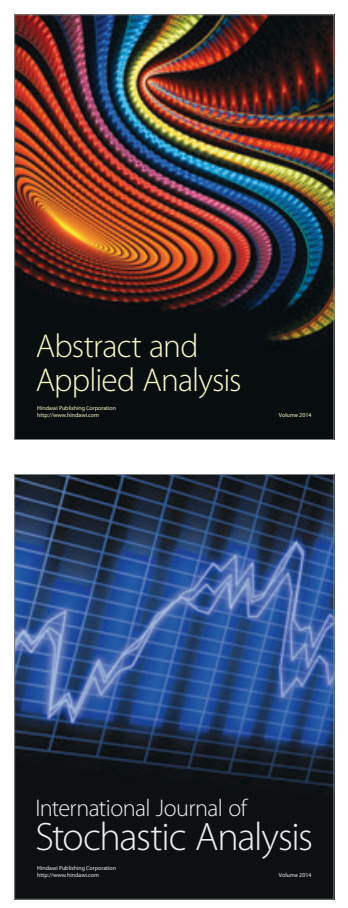

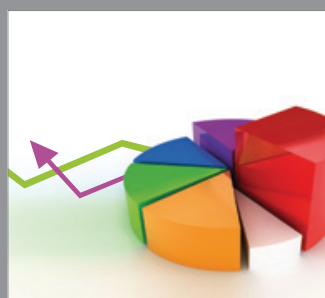

ournal of

Probability and Statistics

Promensencen
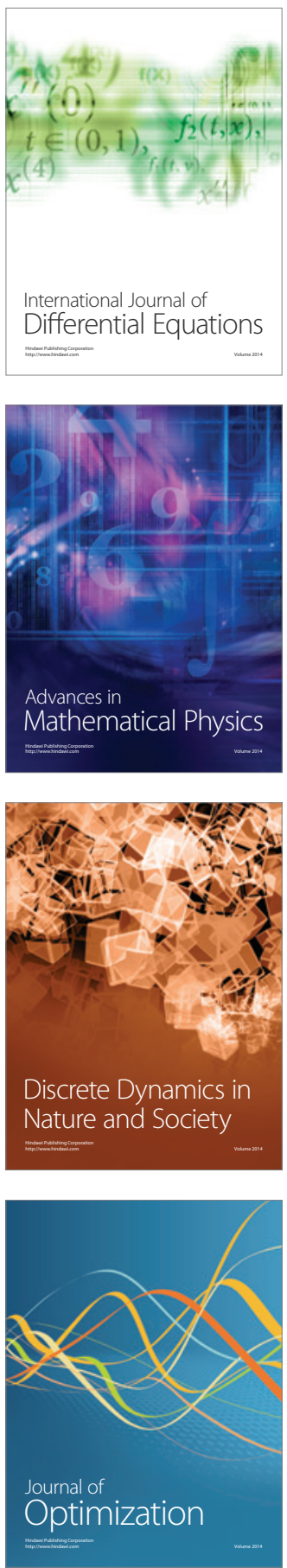